\title{
Une congrégation pentecôtiste congolaise à Montréal
}

Christianisme du Sud, bouture québécoise

\section{Géraldine Mossière}

\section{OpenEdition}

\section{Journals}

Édition électronique

URL : http://journals.openedition.org/assr/17383

DOI : $10.4000 /$ assr. 17383

ISSN : $1777-5825$

Éditeur

Éditions de l'EHESS

Édition imprimée

Date de publication : 1 septembre 2008

Pagination : 195-213

ISBN : 978-2-7132-2191-0

ISSN : 0335-5985

\section{Référence électronique}




\section{Géraldine Mossière}

\section{Une congrégation pentecôtiste congolaise à Montréal Christianisme du Sud, bouture québécoise}

Au Canada comme en France, l'immigration a joué un rôle central dans la construction de la nation. Toutefois, la gestion et l'interprétation des enjeux qui lui sont liés diffèrent selon les pays. Là où le premier évoque l'immigrant et un processus identitaire en perpétuelle négociation, le second tend à parler d'immigrés relevant d'un statut précaire et soumis au seul impératif de l'intégration. En France, en effet, les flux migratoires ont été associés, dans la période récente, au passé colonial et les nouveaux arrivants se sont greffés sur un large bassin de population installé de longue date et nettement majoritaire. Au Canada en revanche, si l'immigration est à l'origine de la constitution du pays autour de deux peuples fondateurs, elle se poursuit aujourd'hui dans le cadre d'une politique démographique et économique planifiée et volontariste. L'organisation sociale du pays repose donc sur une dynamique multi-ethnique marquée, si bien qu'en matière d'intégration, il est désormais commun d'opposer le modèle communautariste et multiculturel canadien au modèle "républicain » et assimilationniste français. Alors qu'en France, les enjeux sociaux et politiques répondent à une logique de sécularisation qui " rend plus illégitime toute forme d'action sociale ou culturelle fondée sur des valeurs religieuses » (Césari, 2004 : 256), le multiculturalisme canadien permet et favorise l'identification individuelle et collective à des groupes dont les marqueurs peuvent se décliner sur une base ethnique, religieuse ou autre. Entre ces deux modèles, l’interculturalisme québécois semble proposer une position intermédiaire (Costa-Lascoux, Mc Andrew, 2005). Ayant récemment acquis pleine compétence en matière de sélection et d'intégration des immigrants, le Québec a développé sa propre philosophie de gestion de l'immigration qui traduit bien le statut de nation qu'il revendique au sein de la confédération canadienne. Ainsi, tout en reconnaissant le rôle essentiel de l'immigration dans son développement démographique ${ }^{1}$, les politiques publiques de la province

1. «Le contexte démographique actuel est marqué par la dénatalité, le vieillissement de la population, une demande accrue de main-d'œuvre et une pénurie prévisible de ressources humaines. C'est pour cela que le Québec reconnaît maintenant plus que jamais l'apport de l'immigration à son développement. " Plan d'action 2004-2007 du Ministère de l'Immigration et des Communautés Culturelles du Québec. Consulté le 17 avril 2008, http://www.micc .gouv.qc.ca/fr/planification/plan-action.html consulté le 17 avril 2008. 
reposent sur un contrat moral qui lie tout nouveau citoyen québécois à une société « de plus en plus diversifiée ", qui néanmoins se « rallie autour de valeurs fondamentales $»^{2}$ : l'égalité entre les sexes, la primauté du français et la séparation entre la religion et l'État. Le système de points qui départage les candidats à l'immigration en évaluant leur capacité d'adaptation à la société d'accueil permet de recruter les immigrants les plus aptes à développer un sentiment d'appartenance à la province, et ainsi à contribuer à la construction de la nation. Bien que cette approche repose sur l'acceptation et l'assimilation de valeurs consensuelles et partagées, la distinction entre l'espace privé et l'espace public y est moins dogmatisée qu'en France, et la dimension communautaire dans l'intégration des nouveaux arrivants est davantage reconnue.

Au cours des trois dernières années, le Québec a accueilli une moyenne de 44400 immigrants (pour une population totale de 7520900 habitants) ${ }^{3}$, conformément à un ensemble d'objectifs de types démographique, économique, linguistique et humanitaire. La gestion politique de cette diversité culturelle s'est toutefois avérée moins porteuse au regard de l'intégration socioéconomique des nouveaux arrivants. En effet, le «temps de purgatoire " ${ }^{4}$ demeure long et ce en dépit du «capital humain » élevé d'une population essentiellement sélectionnée selon des critères d'aptitudes professionnelles et langagières. D'abord limitées aux pays européens, les vagues d'immigration plus récentes proviennent de régions de plus en plus variées qui combinent désormais pluralisme ethnique et religieux. La palette de religions importées par les immigrants ajoute aux traditions catholique et protestante des peuples fondateurs une pluralité de religions perçues comme «exotiques » : sikhisme, islam, hindouisme, mais aussi nouveaux courants chrétiens (charismatiques, pentecôtistes, etc.).

À leur arrivée au Québec, les immigrants s’installent généralement dans la métropole montréalaise où prolifèrent de nouveaux espaces de sociabilité. Les lieux de culte regroupant des minorités ethniques (Africains, Haïtiens ou Philippins) y sont par ailleurs en forte croissance et les religions évangéliques particulièrement dynamiques (Germain et al., 2003). On peut dès lors s'interroger sur les modalités d'inscription de ces "christianismes du sud " au sein d'un champ politique double : la sphère publique québécoise et l'espace transnational. Dans quelle mesure la mobilisation des référents du pays d'origine oriente-t-elle ces congrégations au sein de la dynamique locale-globale dans laquelle l'expérience migratoire les projette ? Comment ces mécanismes modifient-ils les rapports sociaux et structures de pouvoir existants?

2. Informations recueillies sur le site du Ministère de l'Immigration et des Communautés Culturelles du Québec: http://www.quebecinterculturel.gouv.qc.ca/fr/valeurs-fondements/index. html (consulté le 17 avril 2008).

3. http://www.micc.gouv.qc.ca/fr/recherches-statistiques/stats-immigration-recente.html (consulté le 25-04-2008).

4. Délai enregistré avant que le niveau de revenus et d'emploi ne rattrape la moyenne nationale. 
Partant de l'étude d'une Église pentecôtiste congolaise établie à Montréal, nous proposons d'examiner les mécanismes de production identitaire d'une de ces communautés. Cette construction est façonnée par l'univers du discours de la société d'accueil et par le dogme pentecôtiste dont elle reflète les tensions politiques et dialectiques. De fait, ces processus travaillent les catégories de pouvoir et déplacent les frontières qui arbitrent les rapports sociaux au sein de la communauté. Après avoir présenté la Communauté Évangélique de Pentecôte (CEP), nous discuterons les termes du discours qu'elle élabore sur les pratiques langagières, le religieux dans l'espace public et le projet d'expansion transnationale, trois sites discursifs autour desquels l'Église forge son identité. La structure de pouvoir traditionnelle est reconfigurée autour de nouvelles catégories sociales axées sur une grille de références modernes telles que l'éducation et le savoir, qui tend à privilégier les membres jeunes, éduqués et francophones.

Notre étude s'appuie sur une enquête de terrain amorcée en 2002, auprès d'une Église pentecôtiste localisée dans la métropole montréalaise qui rassemble essentiellement des immigrants d'origine africaine et haitienne, les Congolais ${ }^{5}$ étant largement majoritaires. Le corpus de données comprend des observations des cultes dominicaux et de diverses activités religieuses et sociales plus ou moins organisées (rencontres des cellules de quartier, séminaires d'études dispensés par le pasteur, etc.). Des entretiens formels et informels, semi-structurés, ont été également menés avec des membres de la congrégation, d'assiduité variable, ainsi qu'avec des officiants rituels (pasteur, leader de la chorale).

\section{La Communauté Évangélique de Pentecôte (CEP)}

La Communauté Évangélique de Pentecôte est une Église révélée, née de l'initiative de l'actuel pasteur d'origine congolaise. C'est lors d'un voyage touristique au Québec que ce dernier dit avoir ressenti un appel divin lui indiquant que c'était là sa terre de mission. Docteur en théologie, et alors pasteur en Belgique d'une église composée principalement de Blancs, il avait quitté son pays d'origine depuis sept ans quand il décide d'immigrer au Québec en 1991. Un an plus tard, il crée la CEP, une "entreprise religieuse " qui croît à un rythme exponentiel, surtout par le bouche à oreille. Alors que les premières réunions rassemblaient une dizaine de personnes, famille et amis dans le salon de la maison du pasteur, la CEP compte aujourd'hui environ quatre cent trente fidèles qui résident dans divers quartiers de la ville et se réunissent dans un bâtiment acheté dans le MileEnd, un quartier multiethnique et multiconfessionnel de Montréal. Les membres proviennent d'une douzaine de pays d'Afrique principalement francophones (République Démocratique du Congo, Côte-d'Ivoire, Cameroun, Burundi, Rwanda, Bénin), d'autres viennent de l'Angola ou même d'Égypte, mais aussi d'Haïti,

5. Dans l'exposé, le Congo se réfère à la République Démocratique du Congo (RDC). 
sachant qu'une faible minorité de Québécois dits «de souche ${ }^{6}$ fréquentent également l'assemblée. Le profil des fidèles est assez diversifié : familles, personnes divorcées ou veuves, femmes monoparentales. La proportion des moins de trente ans est significative et compte pour un peu moins de la moitié des participants, les femmes demeurant les plus nombreuses. Le site Internet de la CEP présente la communauté comme une "assemblée multiculturelle (...) qui compte parmi ses membres des personnes de divers pays ». En effet, les membres sont généralement des immigrants de première génération installés au Québec depuis moins de vingt ans. Immigrants de type économique ou familial, la plupart des fidèles, notamment haïtiens, gardent l'espoir de rentrer au pays; ils maintiennent généralement des liens, surtout au niveau financier, avec la famille qu'ils y ont laissée.

La majorité des membres de la communauté provenant d'Afrique francophone ont un parcours migratoire plus homogène. Nombre d'entre eux sont des réfugiés politiques qui représentent une main-d'œuvre qualifiée et instruite qui jouissait d'un niveau de vie aisé dans le pays d'origine. C'est la guerre civile qui les a amenés au Québec où leurs qualifications sont rarement validées et où, bien souvent, ils se retrouvent face à un imbroglio administratif qui peut les plonger dans des situations précaires. De façon générale, ils connaissent une réduction brutale de leur niveau de vie et statut social. Quant à la classe des moins de trente ans, ils ont généralement quitté l'Afrique à un très jeune âge soit en migrant directement au Canada, soit en grandissant dans un pays européen francophone avec leurs parents, avant de se rendre au Québec. Quasiment tous les membres de la CEP sont nés dans une tradition chrétienne et la plupart des membres d'origine africaine étaient déjà pentecôtistes avant de s'installer au Québec. Ceux qui décident d' " accepter Jésus-Christ » sont tenus d'abandonner toute forme de "fétichisme". Un rituel de délivrance est alors mené à huis clos, au cours duquel les éventuels objets associés à des pratiques qualifiées d'« occultes » sont détruits. Invoquant les préceptes bibliques, le pasteur critique cette religiosité traditionnelle pour son inefficacité et son incapacité à " produire " succès et effets positifs sur la vie des membres.

La CEP est gérée par une équipe de six pasteurs épaulés par dix diacres dont plusieurs sont des femmes. Le pasteur fondateur dirige l'ensemble de l'organisation, il nomme et forme les leaders rituels et prédicateurs qui officient lors des rituels. Sa légitimité repose sur la reconnaissance par tous de ses dons charismatiques, notamment le don de recevoir et d'interpréter les messages de Dieu.

La CEP est organisée selon le modèle congrégationaliste typique de l'actuel paysage religieux nord-américain. La majorité de son financement provient des offrandes des membres ainsi que de la dîme. C'est une institution locale dont le

6. Sont considérés "de souche » les résidents du Québec qui y sont nés et dont les familles y sont installées depuis au moins deux générations. 
fonctionnement repose sur le volontarisme et l'implication active des membres laïcs dans la vie communautaire. Ces derniers se répartissent au sein de divers ministères : le ministère du protocole est par exemple chargé de l'accueil et du bon déroulement du culte tandis que le ministère des louanges dirige la chorale et anime les rituels. D'autres sont établis en fonction du sexe et de l'âge des membres; diverses activités et thèmes d'étude liés à la société d'accueil y sont abordés par des prédicateurs de la CEP ou par des intervenants extérieurs : le ministère des couples a par exemple invité un juriste à donner un séminaire sur «l'art d'être parent au Québec ». La direction et la gestion de ces organes sont attribuées par le pasteur soit à des membres de sa famille, soit à des personnes ayant un niveau d'éducation élevé. D'autre part, les membres qui résident dans une même zone géographique se regroupent chez l'un ou l'autre particulier, au sein de «cellules de quartier " pour des activités religieuses hebdomadaires. Ces réunions mobilisent des réseaux informels très actifs : par exemple, certains membres se réunissent par téléphone pour prier et chanter ensemble, tôt le matin ou en soirée. Le pasteur chapeaute l'ensemble de l'organisation, il entretient aussi des relations personnelles avec les membres auxquels il rend visite à domicile. Ceux-ci ont également la possibilité de le rencontrer pour obtenir des conseils dans divers domaines de la vie quotidienne, si bien que le pasteur se définit de façon ironique comme un « conseiller spirituel, conseiller matrimonial, conseiller en matière d'emploi, conseiller de la jeunesse, conseiller en funérailles ».

Considéré comme le cœur de l'activité communautaire, le rituel du dimanche réunit tous les membres dans un moment d'intense ferveur religieuse. Toutes les célébrations sont de nature religieuse et aucune fête liée au pays d'origine n'est marquée ou organisée par la congrégation, le pasteur se contentant d'indiquer les événements publics auxquels des membres de la CEP participent, comme les journées interculturelles organisées par la ville de Montréal où certains fidèles tenaient un stand sur la République Démocratique du Congo. Lors des écoles du dimanche, les enfants de la congrégation apprennent les principes et valeurs associés au dogme chrétien mais aucune transmission de connaissance de la langue ou de la culture africaine ou haitienne n'est prévue dans le cadre de l’Église.

Comme la plupart des congrégations religieuses nord-américaines rassemblant des immigrants (Warner, 1998; Menjivar, 2003), la CEP met donc à la disposition des membres des ressources matérielles et psychologiques, soit un soutien pour l'emploi et le logement par exemple, mais aussi des services de conseil et d'encadrement administratif pour les nouveaux arrivants. Le recours à ces ressources reste cependant fort variable : si une majorité de membres s'impliquent très activement dans la communauté, d'autres ne se rendent dans ses locaux qu'à des fins religieuses, principalement le dimanche pour prier et pour recevoir le message divin délivré par le pasteur. 


\section{Des discours de construction identitaire}

L'identité de la CEP se construit autour de trois sites discursifs : les pratiques langagières, le rôle du religieux dans la sphère publique et le projet d'expansion transnational. C'est là que se donnent à voir les univers du discours de la société d'accueil et du pentecôtisme, c'est là aussi que ces discours sont réinterprétés par la communauté religieuse.

\section{Des pratiques langagières dans le culte et dans la communauté}

Le site Internet de la CEP annonce que le français est la «langue principale de l'assemblée » et limite l'usage occasionnel d'autres langues aux "réunions d'adoration ». Même lors des cultes, les adorations et implorations prononcées individuellement dans un rapport voulu intime et exclusif avec Dieu sont exprimées en français. Au Québec, après avoir longtemps reposé sur l'appartenance à l'Église catholique, le mythe fondateur de la nation s'articule aujourd'hui principalement autour de la francophonie de sorte qu'en choisissant d'officier dans la langue du pays d'accueil, la CEP peut prétendre s'y insérer. En effet, les membres de la congrégation présentent l'adoption du français comme une des conditions d'existence au Québec qui modifie leur rapport au pays d'origine, comme l'explique le pasteur :

«Les Congolais qui vivent ici sont des gens déracinés, ça arrive qu'on leur entonne un chant même dans leur langue d'origine, ils n'explosent pas dans la joie habituelle,

à force de fonctionner dans le français et de vivre dans une autre culture... »

Plus qu'un gage d'adhésion au discours du pays d'accueil, l'usage du français transfère pouvoir et statut social à son locuteur puisque les membres l'associent à la classe intellectuelle, instruite et aisée des anciennes colonies européennes. C'est pourquoi certains disent éduquer leurs enfants uniquement en français. En fait, le discours valorisant la langue française participe de la revendication d'une appartenance à une communauté globale francophone. Par exemple, le pasteur est fier d'avoir répertorié sa communauté sur le site francophone Top Chrétien où il rend accessible ses prédications et grâce auquel il dit entretenir une correspondance avec des pentecôtistes de langue française disséminés dans le monde. Les moins de trente ans construisent cette identité transnationale en décrivant leurs performances linguistiques, induites par leurs parcours migratoires transnationaux : ayant quitté le Congo à un très jeune âge, ils ont tous été socialisés en français et certains disent maîtriser difficilement les langues véhiculaires du pays d'origine. Ils disent avoir « la peau congolaise mais la mentalité est plus occidentale qu'autre chose », comme Ludovic, dans la trentaine, titulaire d'une maîtrise en sciences, arrivé en Suisse avec ses parents réfugiés politiques, à l'âge de cinq ans. Il y a grandi et s'y identifie au point d'affirmer : " c'est mon pays, j'ai grandi là-bas, c'était normal que j'y fasse mon service militaire ! ", et d'ajouter : "le Congo, c'est mes racines, j’ai été élevé comme un Congolais! » Ainsi, il parle 
français avec ses parents, et dit maîtriser plus ou moins leur langue vernaculaire. Avec le pasteur, il s'exprime plutôt en lingala (une des langues véhiculaires du Congo) mais, en somme, il est plus à l'aise en français.

Le choix du français comme langue usuelle à la CEP entraîne toutefois une réorganisation des relations sociales, et notamment des rapports intergénérationnels. En effet, en déterminant l'accès des membres à la direction et aux activités de la congrégation, les pratiques langagières réorganisent les rapports de pouvoir au sein même de la communauté. Selon Han (2007) qui a examiné le parcours d'immigrants chinois au sein de communautés religieuses de Toronto, les idéologies des pratiques langagières comptent parmi les outils les plus significatifs pour déterminer et légitimer les systèmes de pouvoir au sein des groupes sociaux. Ainsi, même minoritaires, les membres plus âgés de la CEP, qui ne partagent pas le profil international des plus jeunes, revendiquent l'autorité traditionnellement dévolue aux aînés. Ils demandent alors que certains chants ou certaines rencontres se tiennent dans leur langue vernaculaire à tel point que, lors des cultes, il arrive que des plus jeunes traduisent discrètement les sermons des prédicateurs aux aînés, sans quoi ces derniers en seraient exclus de facto. Les choix et discours relatifs aux modes d'expression forgent par conséquent les logiques d'exclusion et d'inclusion au sein de la communauté, qui confortent la domination symbolique des francophones, une catégorie sociale intimement liée à l'éducation et au niveau socioéconomique, ainsi qu'à la classe d'âge.

\section{De la philosophie de l'Église dans la Cité}

« Je sais que nous irons au ciel un jour, mais nous sommes sur la terre et je suis convaincu que le pasteur a une mission d'encadrement social et quand il la néglige, il a manqué une partie de sa tâche. »

Le pasteur légitime la place de la CEP dans l'espace québécois par la mission sociale qu'il attribue à toute communauté religieuse. La philosophie de l'Église dans la Cité doit contribuer au développement de la communauté en mobilisant les fidèles pour être efficaces et productifs. Ainsi, les activités de la congrégation telles que les cellules de quartier créent des espaces où circulent des informations et des ressources liées à la société d'accueil (informations sur les procédures administratives, offres d'emploi, etc.) Certaines rencontres des ministères sont également consacrées aux problèmes typiques et concrets auxquels sont confrontés les immigrants si bien que les performances rituelles et l'incorporation du message normatif de la congrégation sont interprétées comme une voie de réhabilitation sociale, et considérées comme des vecteurs de mobilité économique individuelle.

Les prédications du pasteur en particulier visent à façonner le comportement citoyen des membres à partir des valeurs de la prospérité économique, ce qui implique une adaptation aux façons de faire de la société d'accueil. Les sermons les informent de certains codes légaux et codes de conduite en vigueur au Québec, en organisant des séminaires ou en consacrant des sermons à des thèmes tels 
que la famille ou le mariage : "comment interpréter le mariage en tant que chrétien, en conformité avec la loi du Québec ». L'éthique ainsi transmise repose sur des valeurs telles que le travail, la discipline et l'accumulation économique qui sont propres à l'éthique protestante (Weber, 1964) dont est issu le pentecôtisme historique et qui s'intègrent favorablement à la société canadienne. La sémantique libérale de l'efficacité capitaliste, du rendement et du savoir traverse la rhétorique de la CEP, à l'instar de cet avertissement d'un prédicateur débutant son sermon : "Aujourd'hui, je vais vous coacher ». En outre, la référence à l'éducation est omniprésente : les conférenciers invités sont présentés selon leur titre universitaire tandis que les prédications du pasteur vantent sans relâche les vertus des emplois qualifiés. Le retour aux études, rendu souvent nécessaire par les procédures de reconnaissance des diplômes du pays d'origine, constitue un des chevaux de bataille du pasteur. D'ailleurs, les membres apprécient davantage les prédications qui privilégient l'approche rationnelle et la crédibilité scientifique considérées comme plus convaincantes. L'une d'entre eux évoque avec enthousiasme le prédicateur belge de l'Église qu'elle fréquentait au Congo :

« Il donne des enseignements de façon que tu vas croire qu'il est chimiste ou géographe ? Il connaît tout ce qui est matière, tout ce qui est vie, il t'explique ça avec la Bible comme ça (elle claque dans ses doigts), il est fort, il est très fort! "

Par ailleurs, les rituels font usage des média et des techniques modernes de communication : l'Église est dotée d'un écran géant où sont affichées les paroles des chants et les messages à la communauté, le matériel de prédication est disponible en DVD pour une somme modique, la communauté a également son propre portail Internet et assure sa présence sur d'autres sites.

Dans un contexte où les institutions et l'imaginaire québécois ont longtemps été dominés par l'Église catholique, le discours de la CEP est façonné par le principe séculier qui règle le mode de gouvernance de la province depuis la Révolution tranquille. En effet, il s'adresse à une population de migrants à laquelle les autorités de la province reconnaissent un rôle fondamental dans son développement, mais dont l'insertion reste un défi majeur. L'intégration de cette rhétorique dominante assure à la CEP une légitimité dans l'espace public québécois. Le pasteur n'hésite pas à blaguer sur le fait que le gouvernement devrait le rémunérer pour vaquer à une partie de sa responsabilité d'accueil des immigrants. Un tel discours modèle toutefois la figure du fidèle idéal autour de nouvelles valeurs typiquement modernes telles que l'éducation ; le pentecôtiste devient un entrepreneur rationnel, mû par un projet de perfectionnement personnel, et animé par la quête d'une grâce divine, elle-même sanctionnée par sa réussite matérielle et sociale.

En fait, l'éthique de la modernité dans laquelle s'inscrit le discours de la $\mathrm{CEP}$ est propre à la société québécoise autant qu'à l'idéologie pentecôtiste. Nous reprenons ici la définition relativiste de la modernité proposée par Jackie Assayag (2005) : une condition globale que les groupes locaux réinterprètent dans une 
pluralité de cadres temporels et spatiaux. Si la CEP calque son discours sur celui de la société d'accueil, elle s'en réapproprie les conditions en les insérant dans le cadre du paradigme chrétien. Bien que la congrégation dise orienter le parcours des membres dans le sens d'un ajustement au contexte moderne et libéral de l'environnement québécois, cet ajustement est présenté et vécu comme une mission visant à l'accomplissement de l'œuvre de Dieu. Ainsi, lors des rituels de témoignage, les membres partagent des expériences migratoires qu'ils assimilent à un cheminement spirituel, ponctué de signaux et d'épreuves divines. Les valeurs mobilisées pour motiver les discours se réfèrent toujours à une grille de références d'ordre religieux.

\section{Le projet d'expansion transnationale}

La création de la CEP se situe dans le vaste mouvement d'Églises indépendantes qui, dans le sillage des missions évangéliques américaines, foisonnent en Afrique depuis le $\mathrm{XIX}^{\mathrm{e}}$ siècle, selon les dynamiques de fusion et de scission typiques des Églises prophétiques. Ainsi, depuis sa création en 1992, la CEP s'est développée selon une double dynamique rhizomique et pyramidale, mobilisant ainsi des réseaux évangéliques transnationaux déployés à divers degrés du local et du global.

Sur la scène québécoise, la communauté entretient des liens plus ou moins étroits avec d'autres Églises pentecôtistes et centres d'éducation biblique, le pasteur donne par exemple des cours dans divers instituts de théologie francophones. Il est régulièrement invité à prêcher au sein d'églises évangéliques de la province, tandis que la CEP reçoit, elle aussi, des pasteurs affiliés à d'autres congrégations, dont certaines regroupent également des immigrants. Lors de la dernière convention qui a rassemblé la communauté quatre jours durant autour du thème "femmes de foi, femmes de l'impossible ", deux des trois prédicateurs étaient des "Québécoises de souche » affiliées à des institutions pentecôtistes, dont l'Église Nouvelle Vie qui compte elle-même plus de trois mille membres issus de trente pays différents. Pour soutenir leur performance rituelle, ces leaders en visite sont souvent accompagnés de membres de leur Église-mère qui se mêlent à l'assemblée de la CEP. Le développement de ces liens est légitimé par un discours d'appartenance commune à une famille évangélique de "frères et sœurs en Christ " qui supplante les allégeances d'origine. Si la majorité des membres de la CEP proviennent du Congo, le pasteur soutient que leurs origines ethniques sont variées, lui-même étant luba alors que d'autres pasteurs associés sont kikongo. Il se qualifie par ailleurs de "décongolisé » et s'attribue une identité internationale qu'il structure au sein d'une communauté chrétienne pensée sur le mode naturalisé de la famille:

" Ici je bâtis un esprit de famille, les gens apprennent à vivre ensemble. Ça a été très difficile pendant la guerre entre le Rwanda et le Congo, nous sommes la seule Église à avoir maintenu les Congolais et les Rwandais autour de la table. Les Congolais 
disent "ils ont envahi notre pays", les Rwandais disent "on ne nous aime pas ici", mais dans mes visites et mes prédications je leur fais comprendre que nous sommes à $18000 \mathrm{~km}$ de chez nous, nous ne pouvons pas mettre dans nos valises des problèmes d'Afrique et les amener ici. L'Église n'est pas congolaise, elle n'est pas rwandaise, l'Église est pour tous ceux qui acceptent de marcher avec Jésus Christ, et les Congolais qui ne voulaient pas marcher avec les Rwandais sont partis et vice versa. »

Ce discours sur l'appartenance collective transforme la vision du monde des membres et reconstruit les rapports de pouvoir autour de nouvelles dichotomies qui structurent le rapport à la société d'accueil. Ainsi, le principe d'exclusion pieux/impie ou sauvé/damné se substitue à la différenciation ethnique ou nationale, comme en témoigne une fidèle d'origine haïtienne :

« Je suis tellement en paix avec moi-même maintenant ces histoires, je vois pas, tel est Blanc ou tel est Noir, maintenant tout le monde, on est des êtres humains au travail [pour l'œuvre de Dieu]. (...) J'ai des amis blancs, mais si tu es chrétien, tu es baptisé, il faut quand même faire la différence. »

La CEP se construit ainsi en référence à une logique évangélique exclusive dont les stratégies de développement ciblent l'ensemble des dénominations protestantes associées aux mouvements de réveil.

De façon plus méthodique, la CEP calque sa croissance sur le profil international de ses membres et instrumentalise leur mobilité géographique au profit d'un projet d'expansion transnational. Au cours des premières années du millénaire, le discours de la congrégation restait focalisé sur la mission de construire la communauté, de la "restaurer » et de la motiver à "se battre " ${ }^{7}$. Désormais, et d'ici à 2010, le pasteur prévoit d'implanter chaque année, sur le sol canadien, une nouvelle église dirigée par un pasteur formé à la CEP. En tant qu'Églisemère, cette dernière offre le premier mois de loyer de façon à encourager la nouvelle assemblée. Une telle politique d'expansion sur le territoire national vise à stabiliser le nombre de fidèles qui ne cesse de fluctuer avec l'émigration de certains vers l'ouest du pays. Le projet est financé par les contributions financières des membres de la CEP (la dîme et les offrandes ponctuelles). Dernièrement, un des pasteurs formé à la CEP a été mandaté dans une église située en banlieue de Montréal, dans le quartier de l'Assomption. Si cette assemblée composée uniquement de Québécois « de souche " a choisi de s'affilier à la CEP c'est, selon le pasteur, parce qu'ils partagent les mêmes principes de croyance, mais aussi en raison de sa propre réputation de prédicateur et de mentor. Le profil de cette église chrétienne n'est plus atypique, ni à Montréal, ni dans les régions québécoises où il arrive que des pasteurs noirs officient dans des églises chrétiennes blanches. À la fin de l'année 2007, la congrégation a inauguré une église à Montréal-nord, une zone relativement défavorisée où réside un grand

7. Chaque année, une thématique nouvelle oriente les prédications et les activités de la communauté. 
nombre de membres de la CEP. Dans l'ouest du pays, une nouvelle communauté s'est implantée à Calgary au début de l'année 2008 tandis qu'un des fils du pasteur a ouvert une église à Vancouver. La trentaine de fidèles qui s'y rassemblent sont surtout des Québécois résidant dans la région, ou des immigrants souhaitant prier en français. L'ensemble de ces églises éparses entretient des liens virtuels par l'intermédiaire de sites Internet évangéliques tels que le site Top Chrétien ${ }^{8}$.

D'autre part, depuis 2005, une vingtaine d'Églises de réveil établies au Congo se sont affiliées à la CEP. Le pasteur présente ces premières initiatives d'expansion internationale comme des projets pilotes qui visent à accomplir une œuvre chrétienne qui se veut englobante et qu'il prévoit de répéter dans d'autres pays d'origine des membres de la congrégation : des aides ponctuelles ont ainsi été envoyées en Haïti. Les termes d'une telle collaboration sont non seulement idéologiques puisque le pasteur forme les leaders de ces Églises au message évangélique et aux techniques de prédication propres à la CEP, mais matériels puisque des ressources physiques circulent également. La communauté a, par exemple, offert des moyens de transport (vélos et motos) à tous les pasteurs qui officient au Congo, elle finance également un programme de microcrédit proposé aux femmes monoparentales d'une de ces assemblées kinoises, la CEP Ngiri Ngiri. D'autres projets sont prévus (alphabétisation, cours de comptabilité domestique, etc.), le tout étant organisé dans le cadre d'une ONG appelée Citadelle de l'espoir.

S'ils visent à assurer sinon la pérennité, du moins la vitalité de la CEP grâce à la participation de nouveaux membres, ces projets d'expansion sont avant tout forgés par le motif pentecôtiste d'accomplissement de la mission de l'Église. À cet égard, la CEP vient d'acquérir un nouvel espace de culte dans un quartier populaire de Montréal où elle dit vouloir desservir la population locale en offrant des services sociaux et ressources matérielles. Un tel discours découle d'un rapport au monde englobant inhérent à l'universalisme protestant et à son prosélytisme missionnaire. En atteste le symbole que s'est donné la communauté : une colombe éclairée par le Livre saint et dont les ailes recouvrent un globe terrestre. Cette idéologie qui se pense universelle, basée sur le monothéisme chrétien, engendre un ethos incorporé par les fidèles et manifesté dans leurs comportements : ayant quitté Vancouver pour poursuivre ses études en Ontario, le fils du pasteur a en effet annoncé qu'il n'ouvrirait pas d'église dans sa nouvelle ville d'adoption, son père confie en riant : «il va le faire j'en suis sûr, je le connais, il pourra pas s'en empêcher!"

8. www.topchretien.com 


\section{La communauté : tensions et dialectique du local et du global}

\section{Idéal de la Nation ou rhétorique de la diversité ?}

Bien que la construction de la nation québécoise repose sur la reconnaissance d'un ensemble de valeurs fondamentales où la francophonie occupe le premier rang, les politiques publiques ainsi que les comportements sociaux s'appuient sur une rhétorique de la diversité et du cosmopolitisme (Meintel et Le Gall, sous presse). Ce discours hégémonique axé sur "l'ouverture ostentatoire à la diversité », soit la "valorisation de l'éclectisme et du cosmopolitisme culturels » (Fridman et Ollivier, 2004) induit une attitude d'ouverture, autant qu'un point de vue moral, envers d'autres cultures (Hannerz, 1996). Selon Appiah (2006), le cosmopolitisme se définit comme une conversation ou un échange entre différentes altérités permettant de s'habituer à l'Autre. En se présentant comme un « espace multiculturel " ${ }^{9}$, la CEP intègre ce discours de promotion de la diversité. Le pasteur se dit d'ailleurs : " préoccupé d'avoir au même endroit, trente, quarante, cinquante nations. Car le monde a changé, il est devenu comme un village, les gens vivent ensemble sans tenir compte des différences culturelles (...). Ici, à Montréal, dans un rayon d'un kilomètre, combien il y a de nations autour? Pourquoi je dois vivre cloisonné dans mon petit univers ? »

Au Québec, le respect du principe de diversité culturelle est garanti par des mesures dites $d$ ' " accommodement raisonnable " qui visent à neutraliser les pratiques non équitables ou de nature discriminatoire (de façon indirecte ou systémique) susceptibles de se développer, sous couvert de relativisme culturel. Les récents et âpres débats que l'application de ces mesures a suscités autant auprès de la classe politique que du public québécois illustrent bien la dialectique dont résulte la construction de la nation québécoise : d'une part un consensus réalisé autour de valeurs partagées par l'ensemble des groupes qui composent la province, et d'autre part la reconnaissance du pluralisme religieux et ethnique induit par les vagues migratoires désormais constitutives du terreau québécois. Le discours de la CEP reflète cette tension, entre insertion au sein d'un espace d'immigration qui se présente comme une nation francophone et séculière d'une part, et son discours valorisant la diversité des origines et des référents d'autre part.

Bien que la congrégation revendique le français comme langue officielle, les rassemblements plus informels tels que les réunions de cellule ou les nuits de prières peuvent emprunter une langue vernaculaire par souci d'inclure une mama plus âgée. Les hymnes, moment d'intense ferveur individuelle et d'effervescence collective se déclinent généralement sur des rythmes africains ou créoles et, à de rares occasions, ils peuvent être chantés dans l'une des langues d'origine des membres, lingala, swahili, kikongo, luba, créole ou français. Le cas échéant, leur

9. Informations tirées du site Internet de la congrégation. 
traduction est projetée sur écran de sorte que, selon le pasteur : « tout le monde trouve sa place, et les gens ne se trouvent pas relégués dans un coin pour dire vous n'avez rien à faire chez nous. » Par ailleurs, la performance rituelle mobilise des dispositions corporelles marquées par la culture d'origine : ainsi, au début de chaque rituel, les visiteurs et nouveaux venus sont cérémonieusement accueillis sous une vague d'applaudissements et de youyous scandés alors que de nombreuses femmes se rendent au culte dominical en libaya ou simplement en pagne, les vêtements traditionnels congolais. Un membre s'explique :

«Il y a des valeurs du passé que je ne peux pas négliger, quand j’adore, quand je célèbre, j'ai des acquis d'un peuple. L'expression d'une Haïtienne qui danse est différente de celle d'une Africaine. Donc nier ma culture est être en contradiction avec moi-même, ma culture est mon identité. Mais ma culture ne doit pas étouffer ma foi, ma foi doit s'exprimer dans ma culture!"

Ces modes d'expression semblent d'autant plus assumés qu'ils sont présentés comme tolérés, voire valorisés par la société d'accueil. Selon le pasteur, « le Québec aussi encourage chaque communauté à promouvoir ses valeurs, pour autant que cela ne dérange personne et que ça ne va pas contre la loi ». C'est ainsi qu'il explique les unions mixtes formées entre immigrants et Québécois ou entre immigrants de différentes origines dans l'assemblée ou, plus simplement, la présence de Blancs qui, selon lui, sont attirés par l'exotisme manifesté lors des célébrations. La CEP s'approprie et affiche l'idéologie dominante du cosmopolitisme par diverses stratégies de visibilité dans l'espace public, telles que l'attribution du titre de pasteur associé à une femme québécoise blanche, ou encore des projets communautaires adressées à la population du quartier : salle de jeux pour les jeunes, distribution de soupe populaire. D'ailleurs à cet égard, la communauté déplore l'attitude sectaire des juifs hassidiques du quartier qui refusent d'organiser des activités sociales conjointes.

Toute expérience migratoire n'induit pas néanmoins un comportement cosmopolite (Roudometof, 2005). En effet, bien que les mouvements liés à la globalisation semblent désormais toucher une gamme de couches sociales de plus en plus large, incluant les plus défavorisées comme les réfugiés politiques (Werbner, 1999), l'ouverture à la diversité requiert des ressources intellectuelles et matérielles réservées à une classe privilégiée et dotée des compétences nécessaires pour négocier avec d'autres systèmes de références (Bauman, 1998 ; Nava, 2002). À la CEP, bien que le cosmopolitisme soit présenté comme une disposition reconnue et désirable, force est de constater que les conditions d'appropriation dépendent de la distribution des ressources matérielles et éducationnelles : compétences dans la langue d'usage dans la société d'accueil, capacité de mobilité physique, accès aux technologies virtuelles. L'adhésion à la rhétorique cosmopolite dominante tend par conséquent à reconfigurer les catégories de pouvoir en redistribuant l'autorité traditionnellement dévolue aux aînés à une catégorie de jeunes, issus de milieux éduqués et aisés, capables de participer à la globalisation et aux phénomènes qui en découlent. 


\section{Plasticité et interprétations locales d'un dogme englobant}

L'affiliation au pentecôtisme lie les fidèles à une communauté imaginaire globale de sorte que les membres de la CEP se définissent comme "enfants de Dieu » et " frères et sœurs en Christ », au sein d'une famille universelle de chrétiens. L'organisation de la congrégation ainsi que son mode liturgique affichent cependant différentes formes d'appropriation locale de ce modèle dogmatique : la mobilisation du corps dans la danse, le style des mélodies et des rythmes, les pratiques vestimentaires et culinaires sont, par exemple, empruntés aux façons de faire du pays d'origine. D'autre part, les expériences vécues au pays d'accueil comme les difficultés d'immigration sont traitées par référence aux idiomes québécois comme la mobilité sociale via les études. Les sermons du pasteur reflètent ces langages :

"J'ai retravaillé ma liturgie et mon homélie : dans une assemblée de Québécois, je ne parlerai pas des rues de Kinshasa, je parlerai des événements qui se sont passés ici, donc je me suis en quelque sorte acclimaté. »

En dépit de la mobilisation de ces dispositions corporelles et cognitives, les membres se reconnaissent plus dans le fondement biblique du culte que dans ses vecteurs d'expression locaux. C'est dans l'interprétation du pentecôtisme, une doctrine universelle relativement plastique et susceptible d'adaptations locales, que peut se comprendre la forme de ce «christianisme du Sud bouture québécoise ». En effet, si l'éthique du pentecôtisme se veut universelle et transnationale, son mode d'expansion est caractérisé par sa flexibilité, et par une certaine capacité d'adaptation aux univers du discours locaux, au service d'un projet prosélyte d'englobement et de l'intime conviction d'être investi d'une mission sociale.

Au sein des cellules de quartier, les réunions hebdomadaires dirigées par des membres désignés par le pasteur donnent lieu à des partages de mets typiques, des échanges de nouvelles du pays, des veillées funéraires pour des proches défunts au pays ; c'est dans ce cadre également que les membres s'autorisent à parler la langue vernaculaire de leur région d'origine. De tels espaces de sociabilité incluent des compatriotes qui ne sont pas toujours affiliés à la CEP, et peuvent déboucher sur l'établissement de réseaux informels de support et d'entraide entre individus partageant une commune expérience d'immigration. Selon l'étude que Ebaugh et Chafetz (2000) ont réalisée auprès de congrégations religieuses aux États-Unis, cette solidarité s'avère essentielle pour l'intégration sociale et économique de certains immigrants pour qui l'institution religieuse constitue le seul espace disponible de sociabilité pour des ressortissants du même pays d'origine. Cette structure en rhizome intègre les cellules de quartier à de nouveaux réseaux qui dépassent le cadre de l'institution religieuse et sont alimentés par des liens sociaux issus du pays d'origine. Ceux-ci se calquent sur les canaux de recrutement qui guident les nouveaux membres à la CEP, le plus efficace étant le bouche à oreille qui transite souvent via un réseau de parents ou d'amis connus au pays. 
Les organismes communautaires publics ou semi-publics chargés d'accueillir les immigrants au Québec dirigent également les nouveaux arrivants vers des associations qui regroupent des individus originaires du même pays, contribuant ainsi à reproduire un sentiment d'appartenance au pays d'origine.

Le discours d'affirmation d'une identité chrétienne universelle s'inscrit dans le mouvement de renouveau religieux contemporain. En Afrique, ce réveil s'accompagne d'une réinterprétation des pratiques traditionnelles dans le cadre du nouveau paradigme moderne importé par les missionnaires chrétiens (LeBlanc, 2003). Une coutume locale telle que la dot est donc revisitée à travers les enseignements des Écritures Saintes et à l'adresse des jeunes de la CEP qui n'ont pas été socialisés au pays, des membres de formation juridique donnent des conférences destinées à exposer « les sens juridique, biblique et culturel de la dot ». Le pasteur souhaite démontrer par là que la dot "n'est pas un prix d'achat, on n'achète pas une personne! ", mai il se dit personnellement en faveur de son maintien. L'éthique globale et moderne du pentecôtisme n'efface pas les références locales et le champ symbolique traditionnel. Celui-ci se perpétue tout en se chargeant d'un sens nouveau, dans des espaces sociaux reconfigurés par la colonisation, la migration ou la domination (Van der Veer, 1996). Cette relecture de la coutume participe d'un effort de reconstruction moderne de la mémoire collective autour de «nouveaux récits » visant à instituer l'histoire postcoloniale de l'Afrique (Mbembe, 1988). Dans des régions d'obédience traditionnellement catholique, le discours importé par les protestants européens et américains est ainsi instrumentalisé et donne à voir l'adhésion des membres aux notions de progrès, de développement économique, de modernisation, ainsi que leur foi dans les promesses de richesse et de succès qui en découlent.

C'est dans cette tension entre universalité et localité qu'il faut situer la stratégie d'expansion internationale de la CEP. Celle-ci reproduit le discours prosélyte pentecôtiste le long des voies de circulation nord-sud ouvertes par les premiers missionnaires. Cependant, comme dans la plupart des Églises d'immigrants établies dans les pays du Nord, ce projet d'englobement se construit autour de l'implantation d'Églises affiliées aux pays d'origine de leurs membres. Ainsi, les églises que la CEP contrôle au Congo existaient déjà antérieurement, mais la plupart ont été mises sur pied par le pasteur qui a installé à leur tête d'anciens étudiants en théologie, issus de son propre réseau universitaire. Si les membres fondateurs de ces églises sont originaires de diverses provinces de la RDC et issus de différents groupes ethniques (luba, kikongo), ils sont généralement tous liés à l'Aumônerie de l'ISTA (Institut Supérieur en Technologies Appliquées) de Kinshasa où ils se sont connus et qui a été fondée par l'actuel pasteur titulaire de la CEP en 1983. Ce dernier est considéré par plusieurs membres comme un père spirituel et pour ceux qui ont perdu leur parent, comme un père de famille. Le siège de Citadelle de l'Espoir se situe sur une parcelle de la commune kinoise de Ngiri Ngiri appartenant au pasteur titulaire. L'ONG est dirigée par la sœur 
du pasteur, en collaboration avec un ami chrétien, membre d'une autre Église de Réveil du quartier, et diplômé en économie. Le développement international de la CEP au Congo repose donc sur la légitimité attribuée à son pasteur fondateur dans le cadre des relations personnelles que ce dernier a tissées avant son départ (collègues universitaires et famille). En outre, l'autorité qui lui est reconnue est favorisée par l'anomie économique et sociale que connaissent les membres des Églises affiliées en RDC, situation qui les place en attente de ressources, surtout matérielles, envoyées par l'Église-mère. Ces activités missionnaires s'accompagnent de la circulation des membres vers le pays d'origine, en particulier des jeunes, dont certains ne sont pas retournés au pays depuis leur départ, vingt ans auparavant. Invoquant des raisons de sécurité et parfois de manque de confort, plusieurs d'entre eux ont néanmoins annulé ou remis leur voyage au pays. À l'inverse, il est rare que les membres des églises affiliées obtiennent les visas nécessaires pour participer aux activités de la CEP au Québec. La mobilité est ainsi réservée aux membres les plus fortunés de la congrégation, mais le témoignage de ces expériences transnationales intègre ceux que le faible niveau de ressources enferme dans le local dans une communauté globale et solidaire. Cette dynamique transnationale transforme les catégories sociales locales et les rapports de pouvoir entre les compatriotes restés au pays et les expatriés. La dialectique locale/globale portée par le dogme pentecôtiste et importée dans les pays d'origine via des projets d'évangélisation déplace le rapport de pouvoir en faveur des Églises d'expatriés établies à l'étranger et de leur vision d'expansion.

\section{Conclusion : Polis pluraliste et dynamiques des frontières}

Le christianisme de la CEP, une congrégation pentecôtiste montréalaise composée d'immigrants africains et haitiens se construit à l'intersection de discours axés sur les pratiques langagières, le rôle de l'Église dans l'espace public et une vision d'expansion transnationale. L'identité de la communauté est ainsi produite, à divers paliers du local et du global, selon des mécanismes forgés par les tensions politiques de la société québécoise dominante où elle s'intègre. La dialectique du discours pentecôtiste oscille entre idéal national et rhétorique du cosmopolitisme, mission d'englobement et stratégie d'adaptation. Ce processus reconfigure la structure de pouvoir et les catégories sociales qui organisent le groupe au profit d'une nouvelle classe de fidèles jeunes, francophones et éduqués affichant un parcours transnational. La référence au pays d'origine se manifeste ponctuellement; elle semble encouragée par la logique cosmopolite de l'univers discursif québécois dans laquelle la CEP prétend s'insérer et dont elle vise à s'approprier la grille de références modernes. L'éducation et le savoir sont valorisés autant par la société d'accueil que par le dogme pentecôtiste. Néanmoins, la CEP se réapproprie le discours de la modernité en recomposant l'histoire collective à partir d'une relecture des pratiques coutumières du pays d'origine. 
Dans le cadre de la mission d'évangélisation qu'elle s'attribue, cette vision prend un sens politique en posant de nouvelles différenciations entre âmes sauvées et âmes égarées.

Illustration d'un mode particulier d'insertion dans le contexte interculturel d'une province d'immigration nord-américaine, l'étude de la CEP met en lumière de nouvelles problématiques : comment comprendre le pluralisme de ces sociétés, sans verser dans la vision d'une authenticité posée comme un a priori. Dans ces espaces alimentés par des groupes d'immigrants aux profils de plus en plus variés, les frontières sociales, loin de disparaître ou de se cristalliser, se déplacent et redéfinissent les périphéries où évoluent de nouvelles marginalités. Les formes d'autorité traditionnelle s'effacent en faveur des jeunes, détenteurs du savoir et considérés comme garants du dynamisme et de l'avenir de la congrégation. L'examen de la participation des personnes plus âgées de la CEP et de leur rôle dans le groupe révélerait probablement des stratégies de résistance à l'encontre de ces nouveaux rapports de pouvoir. À plus long terme, la perspective des membres de la seconde génération dont le nombre va en s'accroissant et dont le parcours est davantage centré sur la société d'accueil, pourrait, à son tour, modifier les références et le mode de construction de la communauté.

Géraldine MOSSIÈRE

Université de Montréal - Département d'anthropologie geraldine.mossiere@umontreal.ca

\section{Bibliographie}

ApPIAH Kwame, 2006, Cosmopolitanism: Ethics in a World of Strangers, New York, Londres, WW Norton.

AsSAYAG Jackie, 2005, La mondialisation vue d'ailleurs : l'Inde désorientée, Paris, Éditions du Seuil.

Bauman Zygmunt, 1998, Globalization: the Human Consequences, New York, Columbia University Press.

Cesari Jocelyne, 2004, L'islam à l'épreuve de l'Occident, Paris, La Découverte.

Costa-Lascoux Jacqueline, Mcandrew Marie, 2005, «Le Québec et la France : deux histoires migratoires ", Santé, Société et Solidarité, 1, pp. 5-18.

Ebaugh Helen Rose, ChafeTz Janet S., 2000, Religion and the New Immigrants, Walnut Creek (CA), Altamira Press.

Fridman Viviana, Ollivier Michèle, 2004, «Ouverture ostentatoire à la diversité et cosmopolitisme. Vers une nouvelle configuration discursive ? ", Sociologie et sociétés, 36, pp. $105-26$.

Germain Annick, Gagnon Julie, Polo Anne-Lise, 2003, L'aménagement des lieux de culte des minorités ethniques : enjeux et dynamiques locales, Montréal, Institut National de la Recherche Scientifique Urbanisation, Culture et Société.

Han Huamei, 2007, Language, Religion and Immigrant Settlement: An Ethnography, Toronto, University of Toronto Press. 
HANnerz Ulf, 1996, Transnational Connections: Culture, People, Places. London, Routledge.

Leblanc Marie-Nathalie, 2003, «Les trajectoires de conversion et l'identité sociale chez les jeunes dans le contexte postcolonial Ouest-africain : les jeunes musulmans et les jeunes chrétiens en Côte-d'Ivoire ", Anthropologie et Sociétés, 27, pp. 85-110.

Мвемве Achille, 1988, Afriques indociles : christianisme, pouvoir et État en société postcoloniale, Paris, Karthala.

Meintel Deirdre, Le Gall Josiane, (sous presse), « Unions mixtes en région et à Montréal : Thème et variations ", in Germain A., Leloup X., Radice M., (éds.), Les nouveaux territoires de l'ethnicité, Montréal, Presses de l'Université Laval.

Nava Mica, 2002, "Cosmopolitan Modernity: Everyday Imaginaries and the Register of Difference ", Theory, Culture and Society, 19, pp. 81-99.

Roudometof Victor, 2005, "Transnationalism, Cosmopolitanism and Glocalization ", Current Sociology, 53, pp. 113-35.

VAn Der Veer Peter, 1996, Conversion to Modernities: The Globalization of Christianity, New York, Routledge.

Weber Max, 1985 [1964], L'éthique protestante et l'esprit du capitalisme, Paris, Plon.

Werbner Pnina, 1999, "Global Pathways. Working class cosmopolitans and the creation of transnational ethnic worlds ", Social Anthropology, 7, pp. 17-35.

\section{Résumé}

Au sein d'une Église pentecôtiste montréalaise, composée d'immigrants d'origine africaine et haïtienne, nous examinons le processus de production identitaire qui s'articule autour de trois sites discursifs: les pratiques langagières, le religieux dans l'espace public et le projet d'expansion transnational. La communauté se construit à divers paliers du local et du global, selon des mécanismes forgés par les tensions politiques qui caractérisent la société québécoise dominante, entre idéal national et rhétorique cosmopolite, et au sein de la dialectique propre au dogme pentecôtiste, entre mission universelle et stratégie d'adaptation. Ce processus reconfigure la structure de pouvoir traditionnelle autour d'une catégorie de membres jeunes, éduqués et francophones, affichant un parcours transnational et mobilisant une grille de références modernes telles que l'éducation et le savoir.

Mots-clés : Église africaine, pentecôtisme, immigration, multiculturalisme, rapports sociaux.

\section{Abstract}

Drawing on ethnographic fieldwork conducted in an African Pentecostal congregation in Montreal, I examine how identity is produced through the analysis of three identity sites: language practices, religion in the public sphere and the mission of transnational expansion. The church's identity is produced at different levels of locall global articulations. The latter are shaped on the one hand by political tensions of the dominant Quebecois society, between the national myth and the rhetoric of cosmopolitism, and on the other hand by pentecostal dialectics, between the mission of universal proselytism and strategies of local adaptation. This process remodels the traditional power structure around a population of young, educated, French speaking members who are mobile across national boundaries and focused on a grid of modern references such as education and knowledge. 
Key words: African Church, Pentecostalism, immigration, multiculturalism, social relationship.

\section{Resumen}

Dentro de una Iglesia pentecostal de Montréal, compuesta por inmigrantes de origen africano y haitiano, examinaremos el proceso de producción identitaria que se articula alrededor de tres espacios discursivos: las prácticas del lenguaje, lo religioso en el espacio público y el proyecto de expansión transnacional. La comunidad se ha construido en diversos planos de lo local y lo global, según mecanismos forjados por las tensiones politicas que caracterizan la sociedad dominante de Quebec, entre el ideal nacional y la retórica cosmopolita, y al interior de la dialéctica propia del dogma pentecostal, entre la misión universal y la estrategia de adaptación. Este proceso reconfigura la estructura de poder tradicional alrededor de una categoría de miembros jóvenes, educados y francófonos, que muestran un recorrido transnacional y moviliza una grilla de referencias modernas como la educación y el saber.

Palabras clave: Iglesia Africana, pentecostalismo, inmigración, multiculturalismo, relaciones sociales. 